TRANSACTIONS OF THE

AMERICAN MATHEMATICAL SOCIETY

Volume 366, Number 2, February 2014, Pages 691-706

S 0002-9947(2013)05880-1

Article electronically published on August 8, 2013

\title{
CONSTRUCTIVE PROJECTIVE EXTENSION OF AN INCIDENCE PLANE
}

\begin{abstract}
MARK MANDELKERN
Abstract. A standard procedure in classical projective geometry, using pencils of lines to extend an incidence plane to a projective plane, is examined from a constructive viewpoint. Brouwerian counterexamples reveal the limitations of traditional pencils. Generalized definitions are adopted to construct a projective extension. The main axioms of projective geometry are verified. The methods used are in accordance with Bishop-type modern constructivism.
\end{abstract}

\section{INTRODUCTION}

In the classical theory of projective geometry, it is a fairly simple matter to extend an incidence plane to a projective plane; a line at infinity is added, and pencils of parallel lines become the points at infinity. A projective plane results; the required projective axioms are satisfied. In a strictly constructive environment, however, such an extension presents difficulties due to the indeterminate nature of arbitrary pencils of lines.

Background. An extension of an incidence plane has been constructed by Heyting [H59] and van Dalen [D63, using intuitionistic methods. This work left open the question of the validity of the projective axiom stating that any two lines have a common point. A recent paper [M13] gave a Brouwerian counterexample to demonstrate that in the Heyting extension the common point axiom is constructively invalid.

New extension method. An extension of an incidence plane will be constructed using less restrictive definitions, admitting the points and lines of nonspecific character that inevitably emerge in a constructive setting. The main axioms of projective geometry, including the axiom that any two lines have a common point, will be verified.

Pencils of lines. Traditionally, a pencil of lines is either a family of parallel lines, or the family of lines passing through a given point. Here we use the intrinsic properties of these specific pencils in adopting a definition which includes pencils with nonspecific properties, such as those that arise in a constructive setting. Points in the extended plane will be based on these generalized pencils.

Virtual lines. A central problem in the construction of a projective extension is the difficulty in determining the nature of an extended line by means of an object in the original plane. A line in the extended plane may or may not contain points of the original plane; it is in general impossible to determine, constructively, which case occurs. This leads to the concept of a virtual line, a set of points which, if

Received by the editors February 2, 2012.

2010 Mathematics Subject Classification. Primary 51A45; Secondary 03F65.

Key words and phrases. Projective extension, incidence plane, constructive mathematics. 
nonvoid, is a line. Virtual lines will be used to construct points and lines in the extended plane.

Bishop-type constructivism. We follow the constructivist principles set out by Errett Bishop. Applying these principles when reworking classical mathematics can have interesting and surprising results. For the distinctive characteristics of Bishoptype constructivism, as opposed to intuitionism or recursive function theory, see BR87.

Axioms. There are various approaches to the constructivization of a classical theory. Bishop's proposal is to find constructive versions of classical theorems, and to give them constructive proofs. Thus we adopt no new axioms; we use only constructive versions of the usual classical axioms for an incidence plane. These axioms are valid on the real plane $\mathbb{R}^{2}$, taking note of Bishop's thesis that "all mathematics should have numerical meaning" 1

Logical setting. This work uses informal intuitionistic logic; it does not operate within a formal logical system. For the origins of modern constructivism, and the disengagement of mathematics from formal logic, see Bishop's Chapter 1, "A Constructivist Manifesto", in [B67] or BB85] see also [S70, R82, and [M85. Concerning the source of misunderstandings in the mathematical community as to the methods and philosophy of constructivism, see B65.

\section{Preliminaries}

We assume an incidence plane $\mathscr{G}=(\mathscr{P}, \mathscr{L})$, with axiom groups $\mathbf{G}$ and $\mathbf{L}$, and definitions, conventions, and results from Section 2 of [M07.

Terminology. Some terminology and notation used here will be slightly different from that used in M07. The line through points $Q$ and $R$ will be denoted by $\overline{Q R}$. When the lines $l$ and $m$ are distinct and have a common point, we will say that they are intersecting (rather than "nonparallel"); the unique common point will be denoted by $l \times m$. Note that the condition intersecting is a primary relation for lines; parallel is its negation. For any set $S$, the term nonvoid, and the expression $S \neq \varnothing$, are applied in the strict sense: an element of $S$ has been constructed; it is not sufficient to prove $\neg(S=\varnothing)$. A distinguished line $l_{0} \in \mathscr{L}$ will be selected.

Constructive mathematics. A characteristic feature of the constructivist program is the meticulous use of the connective "or". To prove " $A$ or $B$ " constructively, it is required that either we prove $A$, or we prove $B$; it is not sufficient to prove the contrapositive $\neg(\neg A$ and $\neg B)$.

To clarify the methods used here, we give examples of familiar properties of the real numbers which are constructively invalid, and also properties which are constructively valid. The following classical properties of a real number $c$ are constructively invalid: "Either $c<0$ or $c=0$ or $c>0$ ", and "If $\neg(c \leq 0)$, then $c>0$ ". The relation $c>0$ is given a strict constructive definition, with far-reaching significance. Then, the relation $c \leq 0$ is defined as $\neg(c>0)$. A constructively valid property of the reals is the Constructive Dichotomy Lemma: If $c<d$, then for any real number $x$, either $x>c$, or $x<d$. This lemma is applied as a constructive substitute for the constructively invalid Trichotomy. For more details, see [B67] or [BB85].

Brouwerian counterexamples. To determine the specific nonconstructivities in a classical theory, and thereby to indicate feasible directions for constructive work,

\footnotetext{
${ }^{1}$ Preface, pages vii-x, in [B67]; reprinted as Prolog, pages 1-3, in [BB85].
} 
Brouwerian counterexamples are used, in conjunction with omniscience principles. A Brouwerian counterexample is a proof that a given statement implies an omniscience principle. In turn, an omniscience principle would imply solutions or significant information for a large number of well-known unsolved problems. This method was introduced by L. E. J. Brouwer $\mathrm{Br} 08$ to demonstrate that use of the law of excluded middle inhibits mathematics from attaining its full significance. Examples will be constructed on the real plane $\mathbb{R}^{2}$. For any real number $t$, the line on $\mathbb{R}^{2}$ with equation $y=t$ will be denoted $l_{t}$.

Omniscience principles may be stated in terms of real numbers; we will have use for the following:

Limited principle of omniscience (LPO). For any real number $c$, either $c=0$ or $c \neq 0$.

Weak limited principle of omniscience (WLPO). For any real number $c$, either $c=0$ or $\neg(c=0)$.

Lesser limited principle of omniscience (LLPO). For any real number $c$, either $c \leq 0$ or $c \geq 0$.

Markov's principle (MP). For any real number $c$, if $\neg(c=0)$, then $c \neq 0$.

A statement is considered constructively invalid if it implies an omniscience principle. Following Bishop, we may at times use the italicized not to indicate a constructively invalid statement. For more information concerning Brouwerian counterexamples and other omniscience principles, see [B67] or [BB85, [M83, [M88], M89, and R02.

\section{PENCILS}

The definition for a pencil of lines will involve the intrinsic properties found in pencils of specific type. This will ensure the inclusion of pencils of unknown type that arise in a constructive environment. The definition will also admit pencils for which no lines have been previously constructed, since this situation often occurs in a constructive setting. We assume the incidence plane $\mathscr{G}=(\mathscr{P}, \mathscr{L})$ as indicated in Section 1 .

\section{Definition 2.1.}

- For any point $Q \in \mathscr{P}$, we define

$$
Q^{*}=\{l \in \mathscr{L}: Q \in l\} .
$$

- For any line $l \in \mathscr{L}$, we define

$$
l^{*}=\{m \in \mathscr{L}: m \| l\} .
$$

- A family of lines $\rho$, of the form $Q^{*}$, where $Q \in \mathscr{P}$, or $l^{*}$, where $l \in \mathscr{L}$, will be said to be a regular pencil.

- A family of lines $\alpha$ will be said to be a pencil if it satisfies these two conditions:

(1) $\alpha$ cannot contain fewer than two lines. That is, $\neg(\alpha=\varnothing)$ and $\neg(\alpha$ is a singleton); equivalently, $\neg(l, m \in \alpha$ implies $l=m)$.

(2) If $l$ and $m$ are distinct lines in $\alpha$ with $l, m \in \rho$, where $\rho$ is a regular pencil, then $\alpha \subset \rho$.

- A pencil of the form $Q^{*}$ will be said to be a point pencil.

- A pencil $\alpha$ with the property that $l \| m$, for any lines $l$ and $m$ in $\alpha$, will be said to be a parallel pencil. 
- A pencil $\alpha$ will be said to be complete if the following condition holds:

(2A) If $l$ and $m$ are distinct lines in $\alpha$ with $l, m \in \rho$, where $\rho$ is a regular pencil, then $\alpha=\rho$.

- A pencil $\alpha$ will be said to be strictly complete if the following condition holds:

(2B) If $\alpha \subset \rho$, where $\rho$ is a regular pencil, then $\alpha=\rho$.

- For any pencil $\alpha$, and any line $l$, we say that $l$ lies outside $\alpha$, written $l \notin \alpha$, if $l \neq m$ for all lines $m \in \alpha$.

- Pencils $\alpha$ and $\beta$ are said to be distinct, written $\alpha \neq \beta$, if there exists a line $l \in \alpha$ such that $l \notin \beta$, or there exists a line $l \in \beta$ such that $l \notin \alpha$.

Notes for Definition 2.1.

(i) Not every parallel pencil is regular. For a Brouwerian counterexample, let $c \in \mathbb{R}$ with $\neg(c=0)$. On $\mathbb{R}^{2}$, set $\alpha=\left\{l \in \mathscr{L}: c \neq 0\right.$ and $\left.l \| l_{0}\right\}$. It is evident that $\alpha$ is a parallel pencil. By hypothesis, $\alpha=m^{*}$ for some line $m \in \mathscr{L}$; thus $m \in \alpha$, and $c \neq 0$. Hence MP results.

For an alternative counterexample, let $c \in \mathbb{R}$ and set $\beta=\{l \in \mathscr{L}: c=0$ and $l \|$ $\left.l_{0}\right\} \cup\left\{l \in \mathscr{L}: c \neq 0\right.$ and $\left.l \| l_{0}\right\}$; now the hypothesis implies LPO. This example may be easily modified so that the Law of Excluded Middle (LEM) results.

(ii) In the definition of a pencil, condition (2A) would not be a suitable substitute for condition (2); this will be indicated by the Brouwerian counterexample in note (i) following Theorem 2.16 ,

(iii) Adding condition (2B) to the definition of a pencil would complicate the construction of pencils in Theorem 2.6 and Theorem 3.4 this will be indicated by Brouwerian counterexamples in the notes following these theorems.

Lemma 2.2. A pencil may be contained in at most one regular pencil.

Proof. Let $\alpha$ be any pencil. First assume that $\alpha \subset Q^{*}$ for some point $Q$, and also $\alpha \subset l^{*}$ for some line $l$. Let $m, n \in \alpha$ and suppose that $m \neq n$; then the lines $m$ and $n$ intersect at $Q$, and are also parallel to $l$, a contradiction. Thus $m=n$, and $\alpha$ contains fewer than two lines, a contradiction.

Now let $\alpha \subset Q^{*}$ and also $\alpha \subset R^{*}$, for points $Q$ and $R$, and suppose that $Q \neq R$. Then for any line $l \in \alpha$, we have $l=\overline{Q R}$, so $\alpha$ contains only one line, a contradiction. Thus $Q=R$, and $Q^{*}=R^{*}$.

Finally, let $\alpha \subset l^{*}$ and also $\alpha \subset m^{*}$, for lines $l$ and $m$. For any lines $n_{1}, n_{2} \in \alpha$, we have $n_{1} \| l$ and $n_{2} \| m$. Suppose that $l$ and $m$ intersect; it follows from Proposition 2.11 of M07 that $n_{1}$ and $n_{2}$ intersect, so $\alpha$ is contained in a point pencil, a contradiction. Hence $l \| m$, so $l^{*}=m^{*}$.

Lemma 2.3. Let $Q \in \mathscr{P}$ and let $l, m \in \mathscr{L}$.

(a) $l \notin Q^{*}$ if and only if $Q$ lies outside $l$.

(b) $m \notin l^{*}$ if and only if $m$ intersects $l$.

Proof. (a) First let $Q \notin l$. If $m \in Q^{*}$, then $Q \in m$, so $l \neq m$. Thus $l \notin Q^{*}$. Now let $l \notin Q^{*}$. Construct the line $n$ so that $Q \in n$ and $n \| l$; then $n \in Q^{*}$, so $l \neq n$. Thus $l$ and $n$ are parallel and distinct, so $Q \notin l$.

(b) First let $m$ intersect $l$, and let $n \in l^{*}$. Then $n \| l$, so $m$ intersects $n$, and $m \neq n$. Thus $m \notin l^{*}$. Now let $m \notin l^{*}$, choose any point $Q \in m$, and draw the line $n$ so that $Q \in n$ and $n \| l$. Then $n \in l^{*}$, so $m \neq n$, and $m$ intersects $n$; thus $m$ intersects $l$. 
Corollary 2.4. Let $\rho$ be any regular pencil, and $l$ any line. If $\neg(l \notin \rho)$, then $l \in \rho$.

Notes for Corollary 2.4.

(i) For an arbitrary pencil, this property would be constructively invalid. For a Brouwerian counterexample, let $c \in \mathbb{R}$ with $\neg(c=0)$, and set $\alpha=\left\{l_{t}: t \in\right.$ $\mathbb{R}$ and $c \neq 0\}$. Then $\neg\left(l_{0} \notin \alpha\right)$, but $l_{0} \in \alpha$ would imply that $c \neq 0$; hence MP results.

The same pencil $\alpha$, together with $\beta=l_{0}^{*}$, may be used to show that the statement "If $\neg(\alpha \neq \beta)$, then $\alpha=\beta$ " is constructively invalid. See, however, Lemma 4.2 ,

(ii) This property will be extended to a wider class of pencils in Theorem 2.17

Corollary 2.5. Let $\alpha$ be any pencil, and $l$ any line. If $\neg(l \notin \alpha)$ and $\alpha \subset \rho$ for some regular pencil $\rho$, then $l \in \rho$.

Theorem 2.6. There exists a complete pencil containing any given lines $l$ and $m$. Define

$$
\begin{aligned}
\varphi_{0} & =\{l, m\}, \\
\varphi_{1} & =\{n \in \mathscr{L}: l \times m \in n\}, \\
\varphi_{2} & =\{n \in \mathscr{L}: n\|l\| m\}, \\
\varphi(l, m) & =\bigcup_{i} \varphi_{i} .
\end{aligned}
$$

Then $\varphi(l, m)$ is a complete pencil containing $l$ and $m$.

Proof. Assume that $\varphi(l, m)$ contains fewer than two lines. Suppose that $l$ intersects $m$, and set $Q=l \times m$; then $\varphi_{1}=Q^{*}$, a contradiction. Thus $l \| m$, and $\varphi_{2}=l^{*}$, a final contradiction. This shows that the assumption is contradictory.

Let $n_{1}$ and $n_{2}$ be distinct lines in $\varphi(l, m)$ with $n_{1}, n_{2} \in \rho$ for some regular pencil $\rho$. In the case $\rho=Q^{*}$ for some point $Q$, we have $n_{1} \times n_{2}=Q$. Each of the lines $n_{1}$ and $n_{2}$ lies in one of the sets $\varphi_{i}$, and the required condition $\alpha=Q^{*}$ is easily verified by considering each of the resulting cases. In the case that $\rho$ is a regular parallel pencil, the verification follows similarly.

Hence $\varphi(l, m)$ is a complete pencil.

\section{Notes for Theorem 2.6.}

(i) It is not assumed that the lines $l$ and $m$ are distinct.

(ii) The stronger conclusion, " $\varphi(l, m)$ is strictly complete", would be constructively invalid. For a Brouwerian counterexample, let $c \in \mathbb{R}$ with $\neg(c=0)$, let $l$ and $m$ be the lines with equations $y=0$ and $y=c x$, and consider the pencil $\varphi(l, m)$. Clearly, $\varphi_{0} \subset O^{*}$, where $O$ is the origin. Let $n \in \varphi_{1}$. Then $l \neq m$, and $l \times m=O$; thus $\varphi_{1}=O^{*}$, and $n \in O^{*}$. Thus $\varphi_{1} \subset O^{*}$. If a line is in $\varphi_{2}$, then $l \| m$, so $c=0$, a contradiction; thus $\varphi_{2}=\varnothing$. This shows that $\varphi(l, m) \subset O^{*}$. By hypothesis, $\varphi(l, m)=O^{*}$, so $m_{0} \in \varphi(l, m)$, where $m_{0}$ is the $y$-axis. It follows that $m_{0} \in \varphi_{1}$, and thus $c \neq 0$. Hence MP results.

(iii) When $l \neq m$, the Heyting "p.point" is defined in [H59] by $\mathfrak{P}(l, m)=\{n \in$ $\mathscr{L}: n \cap l=l \cap m$ or $n \cap m=l \cap m\}$. Clearly, $\varphi(l, m) \subset \mathfrak{P}(l, m)$, and $\neg(\varphi(l, m) \neq$ $\mathfrak{P}(l, m))$. However, equality here would be constructively invalid. For a Brouwerian counterexample, let $c \in \mathbb{R}$ and let $l$ and $m$ be the lines with equations $y=0$ and $y=1-c x$. Consider the line with equation $y=2-2 c x$; LPO results. 
Many other results in the present paper also have analogues in [H59]. However, the basic definitions differ in quite fundamental ways, so a detailed comparison of the two approaches is not feasible.

(iv) This method will be extended in Theorem 3.4 .

\section{Lemma 2.7.}

(a) A complete pencil $\alpha$ is a point pencil if and only if $\alpha \cap l^{*} \neq \varnothing$ for every regular parallel pencil $l^{*}$.

(b) Point pencils $Q^{*}$ and $R^{*}$, if distinct, have the line $\overline{Q R}$ in common.

Proof. (a) The necessity is the parallel postulate. For the converse, choose any intersecting lines $m_{1}$ and $m_{2}$, and select lines $n_{1} \in \alpha \cap m_{1}^{*}$ and $n_{2} \in \alpha \cap m_{2}^{*}$. It follows that $n_{1}$ intersects $n_{2}$, so $\alpha=Q^{*}$, where $Q=n_{1} \times n_{2}$.

(b) We may select a line $l \in R^{*}$ such that $l \notin Q^{*}$. Then $Q \notin l$ and $R \in l$, so $Q \neq R$. Thus $\overline{Q R}$ is a line common to both pencils.

Lemma 2.8. For any given complete pencil $\alpha$, the statement “ $\neg(\alpha=\rho)$ for every regular pencil $\rho$ ", is contradictory. However, the statement "Every strictly complete pencil is either a point pencil or a regular parallel pencil" is constructively invalid.

Proof. Assume the first statement. Let $l, m \in \alpha$, and suppose that $l \neq m$. If $l$ intersects $m$, with $Q=l \times m$, then $\alpha=Q^{*}$, a contradiction. Thus $l \| m$, so $\alpha=l^{*}$, also a contradiction. Hence $l=m$. This shows that $\alpha$ contains fewer than two lines, a final contradiction.

For a Brouwerian counterexample to the second statement, let $c \in \mathbb{R}$, let $l$ and $m$ be the lines with equations $y=0$ and $y=1-c x$, and set $\beta=\varphi(l, m)$ using the construction of Theorem 2.6. The pencil $\beta$ is strictly complete; apply the hypothesis to $\beta$, and LPO results.

Theorem 2.9. Let $Q^{*}$ be any point pencil, and $\beta$ any complete pencil. Then $\neg\left(Q^{*} \cap \beta=\varnothing\right)$. However, the stronger conclusion, " $Q^{*} \cap \beta \neq \varnothing$ ", is constructively invalid.

Proof. Assume that $Q^{*} \cap \beta=\varnothing$. Let $l, m \in \beta$ and suppose that $l \neq m$. Suppose further that $l$ intersects $m$, and set $R=l \times m$; then $\beta=R^{*}$. Now suppose even further that $R \neq Q$; then $\overline{Q R} \in Q^{*} \cap \beta$, a contradiction. Thus $R=Q$, and $\beta=Q^{*}$, a contradiction. Thus $l \| m$ and $\beta=l^{*}$. Since the parallel postulate provides a line in $l^{*}$ passing through $Q$, this is a contradiction. Thus $l=m$. This shows that $\beta$ contains fewer than two lines, a final contradiction. Hence $\neg\left(Q^{*} \cap \beta=\varnothing\right)$.

For a Brouwerian counterexample to the stronger conclusion, let $c \in \mathbb{R}$ and take $Q$ at the origin in $\mathbb{R}^{2}$. Let $l$ and $m$ be the lines with equations $y=1$ and $y=1+c-c x$, and let $\beta=\varphi(l, m)$ be the complete pencil constructed using Theorem 2.6. Set $R=(1,1)$. Note that if $c=0$, then $\beta=l^{*}$, so the $x$-axis $l_{0}$ is the unique line common to $Q^{*}$ and $\beta$. On the other hand, if $c \neq 0$, then $\beta=R^{*}$, and $\overline{Q R}$ is the unique common line. By hypothesis, $Q^{*}$ and $\beta$ have a common line $n$; either $n \neq l_{0}$, or $n \neq \overline{Q R}$. It follows that either $\neg(c=0)$, or $c=0$. Hence WLPO results.

Applications of this theorem will be by way of the following:

Corollary 2.10. If $\alpha$ is a complete pencil with the property that $\alpha \cap \beta=\varnothing$ for some complete pencil $\beta$, then $\alpha$ is a parallel pencil. 
The definition of a pencil includes multiple pencils which will represent the same point in the projective extension. Thus we adopt the following:

\section{Definition 2.11.}

- Pencils $\alpha$ and $\beta$ will be said to be equivalent, written $\alpha \approx \beta$, if for any regular pencil $\rho$, the following condition is satisfied: $\alpha \subset \rho$ if and only if $\beta \subset \rho$.

- The pencils $\alpha$ and $\beta$ are said to be nonequivalent if $\neg(\alpha \approx \beta)$.

- With regard to the resulting equivalence relation $\approx$, the equivalence class containing the pencil $\alpha$ will be denoted $\bar{\alpha}$.

Theorem 2.12. Let $\alpha$ and $\beta$ be any pencils.

(a) If $\alpha$ and $\beta$ have two distinct lines in common, then $\alpha \approx \beta$. Nonequivalent pencils have at most one line in common.

(b) If $\alpha$ is a parallel pencil, and $\alpha \approx \beta$, then $\beta$ is also a parallel pencil.

(c) If $\neg(\alpha \neq \beta)$, then $\alpha \approx \beta$. Conversely, if $\alpha \approx \beta$, with $\alpha$ complete and $\beta$ strictly complete, then $\neg(\alpha \neq \beta)$.

Proof. (a) Let $l$ and $m$ be lines common to $\alpha$ and $\beta$, with $l \neq m$. If $\alpha \subset \rho$ for some regular pencil $\rho$, then $l, m \in \rho$, so $\beta \subset \rho$. Thus $\alpha \approx \beta$.

(b) Let $l, m \in \beta$, and suppose that $l$ and $m$ intersect at some point $Q$. Then $\beta \subset Q^{*}$, so also $\alpha \subset Q^{*}$. For any lines $u, v \in \alpha$, we have $u \| v$. If $u \neq v$, then $\alpha \subset u^{*}$, contradicting Lemma 2.2. Thus $u=v$, and $\alpha$ contains fewer than two lines, a contradiction; hence $l \| m$.

(c) Let $\neg(\alpha \neq \beta)$. Let $\alpha \subset \rho$, let $l \in \beta$, and suppose that $l \notin \rho$. Then $l \notin \alpha$, so $\alpha \neq \beta$, a contradiction. Thus $\neg(l \notin \rho)$, so by Corollary 2.4 we have $l \in \rho$. Thus $\beta \subset \rho$. This shows that $\alpha \approx \beta$.

Now let $\alpha \approx \beta$, with $\alpha$ complete and $\beta$ strictly complete, and assume that $\alpha \neq \beta$. Suppose that $\alpha=\rho$ for some regular pencil $\rho$; then $\alpha \subset \rho$, so $\beta \subset \rho$, and $\beta=\rho$, a contradiction. Thus $\neg(\alpha=\rho)$ for all regular pencils $\rho$. By Lemma 2.8, this is a contradiction. Hence $\neg(\alpha \neq \beta)$.

\section{Lemma 2.13.}

(a) Let $\alpha$ be any pencil, and $\rho$ a regular pencil. Then $\alpha \approx \rho$ if and only if $\alpha \subset \rho$.

(b) There exists at most one regular pencil in any given equivalence class of pencils.

Proof. In (a), let $\alpha \subset \rho$; then it follows from Lemma 2.2 that $\alpha \approx \rho$. The converse is immediate. Now (b) follows from (a).

Theorem 2.14. Let $\alpha$ and $\beta$ be parallel pencils.

(a) If $\alpha$ and $\beta$ have a line in common, then $\alpha \approx \beta$. Nonequivalent parallel pencils have no common lines.

(b) If $\alpha$ and $\beta$ have a line in common, and are strictly complete, then $\alpha=\beta$.

Proof. Select a common line $l$; then $\alpha \subset l^{*}$ and $\beta \subset l^{*}$. In (a), it follows from Lemma 2.13 that $\alpha \approx \beta$. In (b), we have $\alpha=l^{*}$ and $\beta=l^{*}$; thus $\alpha=\beta$.

Definition 2.15. For any pencil $\alpha$, we define

$$
\alpha^{\prime}=\bigcup\{\beta: \beta \in \bar{\alpha}\} .
$$

After verification in the next theorem, the pencil $\alpha^{\prime}$ will be called the full pencil in the equivalence class $\bar{\alpha}$. A pencil $\alpha$ will be said to be prime if $\alpha=\alpha^{\prime}$. 
Theorem 2.16. The union $\alpha^{\prime}$ of all pencils in an equivalence class $\bar{\alpha}$ is a strictly complete pencil, equivalent to the pencils in the class.

The pencil $\alpha^{\prime}$ is unique in this limited sense: If $\beta$ is any complete pencil in the equivalence class $\bar{\alpha}$, then $\neg\left(\beta \neq \alpha^{\prime}\right)$. However, the statement "If $\beta$ is any strictly complete pencil in $\bar{\alpha}$, then $\beta=\alpha^{\prime}$ " is constructively invalid.

If $\bar{\alpha}$ contains a regular pencil $\rho$, then $\alpha^{\prime}=\rho$.

Proof. (i) To show that $\alpha^{\prime}$ is a pencil, let $l$ and $m$ be distinct lines in $\alpha^{\prime}$ with $l, m \in \rho$ for some regular pencil $\rho$, and select pencils $\beta_{1}, \beta_{2} \in \bar{\alpha}$ with $l \in \beta_{1}$ and $m \in \beta_{2}$.

Set $\beta=\beta_{1} \cup \beta_{2}$. To show that $\beta$ is a pencil, let $n_{1}$ and $n_{2}$ be distinct lines in $\beta$ with $n_{1}, n_{2} \in \sigma$ for some regular pencil $\sigma$. If $n_{1}$ and $n_{2}$ both belong to $\beta_{1}$, then $\beta_{1} \subset \sigma$, so also $\beta_{2} \subset \sigma$, and $\beta \subset \sigma$. Similarly for $\beta_{2}$. Thus we may assume that $n_{1} \in \beta_{1}$ and $n_{2} \in \beta_{2}$. Let $n \in \beta$; we may assume that $n \in \beta_{1}$. Suppose that $n \notin \sigma$; it follows that $n \neq n_{1}$, with $n, n_{1} \in \beta_{1}$. Then $\beta_{1} \subset \sigma$, so also $\beta_{2} \subset \sigma$, and $\beta \subset \sigma$, so $n \in \sigma$, a contradiction. Thus $n \in \sigma$. This shows that $\beta \subset \sigma$. Hence $\beta$ is a pencil. Clearly, $\beta \approx \beta_{1}$, so $\beta \in \bar{\alpha}$.

Since $l$ and $m$ are distinct lines in $\beta$ with $l, m \in \rho$, it follows that $\beta \subset \rho$. For any pencil $\gamma \in \bar{\alpha}$, we have $\gamma \approx \beta$, so $\gamma \subset \rho$; thus $\alpha^{\prime} \subset \rho$. Hence $\alpha^{\prime}$ is a pencil.

(ii) Let $\rho$ be a regular pencil with $\alpha \subset \rho$. Then $\gamma \subset \rho$ for all pencils $\gamma \in \bar{\alpha}$, so $\alpha^{\prime} \subset \rho$. Hence $\alpha^{\prime} \approx \alpha$.

(iii) If $\alpha^{\prime} \subset \rho$ for some regular pencil $\rho$, then it follows from Lemma 2.13 that $\alpha^{\prime} \approx \rho$, so $\rho \in \bar{\alpha}$, and $\rho \subset \alpha^{\prime}$; thus $\alpha^{\prime}=\rho$. This shows that $\alpha^{\prime}$ is a strictly complete pencil.

(iv) If $\beta$ is a complete pencil in the equivalence class $\bar{\alpha}$, then it follows from Theorem 2.12 that $\neg\left(\beta \neq \alpha^{\prime}\right)$.

(v) For a Brouwerian counterexample involving the statement in quotes, let $c \in \mathbb{R}$, let $m$ be the line on $\mathbb{R}^{2}$ with equation $y=1-c x$, and set $\alpha=\varphi\left(l_{0}, m\right)$. If $\alpha \subset n^{*}$ for some line $n$, then $c=0$ and $\alpha=l_{0}^{*}=n^{*}$. If $\alpha \subset R^{*}$ for some point $R$, with $R=(d, e)$, then $e=0$ and $1-c d=0$, so $c \neq 0$ and $\alpha=(1 / c, 0)^{*}=R^{*}$.

Now consider the family of lines $\beta=\left\{l \in \mathscr{L}: c=0\right.$ and $\left.l \| l_{0}\right\} \cup\{l \in \mathscr{L}: c \neq$ 0 and $(1 / c, 0) \in l\}$. It is clear that $\beta$ is a pencil. Let $\beta \subset n^{*}$ for some line $n$; then $c=0$ and $\beta=l_{0}^{*}=n^{*}$. Now let $\beta \subset R^{*}$ for some point $R=(d, e)$. Suppose that $|c|<1 /(|d|+1)$. Suppose further that $c \neq 0$; then $\beta=(1 / c, 0)^{*}$. It follows that $R=(1 / c, 0)$, so $d=1 / c$ and $|d|>|d|+1$, an absurdity. Thus $c=0$, and $\beta=l_{0}^{*}$, a contradiction. This shows that $|c| \geq 1 /(|d|+1)$, so $c \neq 0$, and $\beta=(1 / c, 0)^{*}=R^{*}$.

Thus $\beta$ is strictly complete, and $\beta \approx \alpha$. By hypothesis, $\beta=\alpha^{\prime}$. Since $l_{0} \in \alpha \subset \alpha^{\prime}$, it follows that $l_{0} \in \beta$, and LPO results.

(vi) If $\rho \in \bar{\alpha}$ for some regular pencil $\rho$, then $\alpha^{\prime} \approx \rho$, so by Lemma 2.13 we have $\alpha^{\prime} \subset \rho$. Thus $\alpha^{\prime}=\rho$.

Notes for Theorem 2.16.

(i) The proof shows that the union of any two equivalent pencils is also a pencil, equivalent to the given pencils. However, the union of two complete equivalent pencils need not be complete. For a Brouwerian counterexample, let $c \in \mathbb{R}$ with $\neg(c=0)$. On $\mathbb{R}^{2}$, set $\alpha=\left\{l_{0}\right\} \cup\left\{l_{t}: t \in \mathbb{R}\right.$ and $\left.c \neq 0\right\}$ and $\beta=\left\{l_{1}\right\} \cup\left\{l_{t}: t \in\right.$ $\mathbb{R}$ and $c \neq 0\}$; these pencils are complete and equivalent. The hypothesis applied to $\alpha \cup \beta$ results in MP. This is one of the facts concerning complete pencils that necessitates the more inclusive definition of a pencil adopted in Definition 2.1.

(ii) The union of two strictly complete equivalent pencils is strictly complete. 
(iii) The counterexample in the proof of the theorem involves an equivalence class containing no regular pencil. On the other hand, it follows from Lemma 2.8 that the statement "If $\rho$ is any regular pencil, then $\neg\left(\alpha^{\prime}=\rho\right)$ " is contradictory.

Theorem 2.17. Let $\alpha$ be a prime pencil, and $l$ any line. If $\neg(l \notin \alpha)$, then $l \in \alpha$.

Proof. Set $\beta=\alpha \cup\{l\}$. To show that $\beta$ is a pencil, let $m_{1}$ and $m_{2}$ be distinct lines in $\beta$ with $m_{1}, m_{2} \in \rho$ for some regular pencil $\rho$. Either both lines are in $\alpha$, or one line is $l$.

In the first case, since $\alpha$ is a pencil we have $\alpha \subset \rho$. It follows from Corollary 2.5 that $l \in \rho$; thus $\beta \subset \rho$.

In the second case, we may say that $m_{1} \in \alpha$ and $m_{2}=l$. Let $n$ be any line in $\alpha$, and suppose that $n \notin \rho$. If $\alpha$ were a regular pencil, then by Corollary 2.4 we would have $m_{2}=l \in \alpha$, and it would follow that $\alpha \subset \rho$, so $n \in \rho$, a contradiction. Thus $\neg(\alpha$ is regular $)$, and by Lemma 2.8 this is contradictory. Thus $n \in \rho$. This shows that $\alpha \subset \rho$, and thus $\beta \subset \rho$. Hence $\beta$ is a pencil.

To show that $\beta$ is equivalent to $\alpha$, let $\alpha \subset \rho$ for some regular pencil $\rho$. It follows from Corollary 2.5 that $l \in \rho$, and this shows that $\beta \subset \rho$. Thus $\beta \approx \alpha$. Since $\alpha$ is a prime pencil, it follows that $\beta \subset \alpha$, and therefore $l \in \alpha$.

\section{VIRTUAL LINES}

Consider the following classical situation: If a line $L$ in the extended plane contains a proper point, then the set $S$, of all proper points on $L$, is a line in the original plane. However, if $L$ is the line at infinity, then the set $S$ is void. Constructively, we will not know in general which is the case. Thus we adopt the following:

\section{Definition 3.1.}

- A set $p$ of points in $\mathscr{P}$ will be said to be a virtual line, or $v$-line, if it satisfies this condition: If $p$ is nonvoid, then $p$ is a line.

- The family of all v-lines will be denoted $\mathscr{V}$.

- The v-lines $p$ and $q$ will be said to be distinct, written $p \neq q$, if they satisfy these two conditions:

(1) $\neg(p=q)$.

(2) If $p, q \in \mathscr{L}$, then $p \neq q$ in the sense of distinct lines in $\mathscr{L}$.

- The expression $p \neq \varnothing$ will imply that the v-line $p$ is nonvoid, and thus $p$ is a line.

- When we write $p \times q=Q$, or $p \| q$, this will imply that $p$ and $q$ are lines.

The notion of a $v$-line also arises in connection with pencils. Theorem 2.12 shows that nonequivalent pencils have at most one line in common, and Theorem 2.14 shows that nonequivalent parallel pencils have no common lines. Thus the family of lines common to two nonequivalent pencils may consist of a single line, or it may be void; constructively, it is in general unknown as to which alternative holds.

Definition 3.2. For any nonequivalent pencils $\alpha$ and $\beta$, we define

$$
\alpha \sqcap \beta=\{Q \in \mathscr{P}: Q \in l \in \alpha \cap \beta \text { for some } l \in \mathscr{L}\} .
$$

The set of points $\alpha \sqcap \beta$ will be called the core of the pair $\alpha, \beta$.

Lemma 3.3. The core $\alpha \sqcap \beta$, where $\alpha$ and $\beta$ are any nonequivalent pencils, is a $v$-line. 
Proof. Set $p=\alpha \sqcap \beta$, and let $p \neq \varnothing$. Construct a point $R \in p$ and a line $m$ such that $R \in m \in \alpha \cap \beta$; clearly $m \subset p$. For any point $Q \in p$, we have $Q \in l \in \alpha \cap \beta$ for some line $l$; it follows from Theorem 2.12 that $l=m$, so $Q \in m$. This shows that $p \subset m$. Hence $p=m$, so $p$ is a line.

Theorem 3.4. Let $p$ and $q$ be any v-lines. Define

$$
\begin{aligned}
\varphi_{0} & =\{p, q\} \cap \mathscr{L}, \\
\varphi_{1} & =\left\{l \in Q^{*}: p \times q=Q\right\}, \\
\varphi_{2} & =\left\{l \in p^{*}: p \| q\right\}, \\
\varphi_{3} & =\left\{l \in p^{*}: p \neq \varnothing, q=\varnothing\right\} \cup\left\{l \in q^{*}: q \neq \varnothing, p=\varnothing\right\}, \\
\varphi_{4} & =\left\{l \in l_{0}^{*}: p=q=\varnothing\right\}, \\
\varphi(p, q) & =\bigcup_{i} \varphi_{i} .
\end{aligned}
$$

Then $\varphi(p, q)$ is a complete pencil.

To aid in the proof, and for later use, we have first:

Lemma 3.5. In Theorem 3.4,

(a) If $p \times q=Q$, then $\varphi(p, q)=Q^{*}$.

(b) If $p \| q$, then $\varphi(p, q)=p^{*}$.

(c) If there exists a line $l$ in $\varphi_{2} \cup \varphi_{3} \cup \varphi_{4}$, then $\varphi(p, q)=l^{*}$.

(d) If $p=\varnothing$ or $q=\varnothing$, then $\varphi(p, q)$ is a parallel pencil.

Proof. The first three properties are immediate. For (d), let $q=\varnothing$, and let $l, m \in$ $\varphi(p, q)$; then $l \in \varphi_{0} \cup \varphi_{3} \cup \varphi_{4}$. If $l \in \varphi_{0}$, then $l=p$, so $p$ is nonvoid, and $\varphi_{3}=p^{*}$. Since $m \in \varphi_{0}$ or $m \in \varphi_{3}$, it follows that $m \| l$. If $l$ lies in $\varphi_{3}$ or $\varphi_{4}$, then (c) applies.

Proof of Theorem 3.4. Assume that " $l, m \in \varphi(p, q)$ implies $l=m$ ". Suppose that one of the v-lines, say $p$, is nonvoid. Now suppose that $q \neq \varnothing$, and then suppose further that $p$ intersects $q$, with $Q=p \times q$. Then $\varphi(p, q)=Q^{*}$, a contradiction. Thus $p \| q$, so $\varphi(p, q)=p^{*}$, a contradiction. Thus $q=\varnothing$, and $\varphi(p, q)=p^{*}$, a contradiction. Thus both v-lines are void, so $\varphi(p, q)=l_{0}^{*}$, contradicting the assumption. This shows that $\varphi(p, q)$ cannot contain fewer than two lines.

Let $l, m \in \varphi(p, q)$ with $l \neq m$. First let $l$ intersect $m$, with $Q=l \times m$; then $l, m \in \varphi_{0} \cup \varphi_{1}$. In the first case, where $l, m \in \varphi_{0}$, it follows that $p \times q=Q$, so $\varphi(p, q)=Q^{*}$. In the other three cases, $\varphi_{1} \neq \varnothing$, and again $\varphi(p, q)=Q^{*}$. Now let $l \| m$. Using part (c) of the lemma we may assume that $l, m \in \varphi_{0} \cup \varphi_{1}$. Since $\varphi_{1}=\varnothing$, we may say that $l=p$ and $m=q$. Now $\varphi(p, q)=\varphi_{2}=p^{*}=l^{*}$.

Hence $\varphi(p, q)$ is a complete pencil.

\section{Notes for Theorem 3.4 .}

(i) It is not assumed that the v-lines $p$ and $q$ are distinct.

(ii) The stronger conclusion, " $\varphi(p, q)$ is strictly complete", would be constructively invalid. For a Brouwerian counterexample, let $c \in \mathbb{R}$ with $\neg(c=0)$, set $p=\{(t, 0): t \in \mathbb{R}$ and $c \neq 0\}$, and set $q=\{(0, t): t \in \mathbb{R}$ and $c \neq 0\}$. Then $\varphi(p, q) \subset(0,0)^{*}$, but $\varphi(p, q)=(0,0)^{*}$ would imply MP. 
The definition of a $v$-line includes multiple v-lines which will form the basis for the same line in the projective extension. Thus we adopt the following:

\section{Definition 3.6.}

- The v-lines $p$ and $q$ will be said to be equivalent, written $p \approx q$, if $\neg(p \neq q)$.

- After verification in Theorem 3.8 the equivalence class containing the v-line $p$ will be denoted $\bar{p}$.

- For any v-line $p \in \mathscr{V}$, we define

$$
p^{\prime}=\bigcup\{q: q \in \bar{p}\} .
$$

- The v-line $p$ will be said to be prime if $p=p^{\prime}$.

- The family of all prime v-lines will be denoted $\mathscr{V}^{\prime}$.

Lemma 3.7. For any v-lines $p$ and $q$, the following conditions are equivalent:

(a) $p \approx q$.

(b) For any line $l \in \mathscr{L}, p \subset l$ if and only if $q \subset l$.

Proof. Let $p \approx q$, and let $p \subset l$ for some line $l$. Let $Q \in q$, and suppose that $Q \notin l$; then $\neg(Q \in p)$, so $\neg(p=q)$. If $p \in \mathscr{L}$, then $p=l$, so $Q \notin p$; thus $p$ and $q$ are distinct as lines. This shows that $p$ and $q$ are distinct as v-lines, a contradiction; thus $Q \in l$. Hence $q \subset l$.

Let condition (b) hold, and assume that $p \neq q$. Under these conditions, it is clear that the two v-lines cannot both be nonvoid. Suppose that one of the v-lines, say $p$, is nonvoid; it follows that $q$ is void. Select any two distinct lines; since $q$ is contained in both lines, condition (b) implies that the line $p$ is contained in both lines, an absurdity. Thus both v-lines are void. Now $p=q$, a contradiction. This shows that $\neg(p \neq q)$, so $p \approx q$.

Theorem 3.8. The relation $\approx$ on the family $\mathscr{V}$ of $v$-lines is an equivalence relation. The union $p^{\prime}$ of all v-lines in an equivalence class $\bar{p}$ is a v-line, equivalent to the $v$-lines in the class.

Proof. It follows from the lemma that $\approx$ is an equivalence relation. Let $p^{\prime} \neq \varnothing$, and select a v-line $r \in \bar{p}$ with $r \neq \varnothing$; thus $r$ is a line. For any $\mathrm{v}$-line $q \in \bar{p}$, it follows that $q \subset r$. Thus $p^{\prime} \subset r$, so $p^{\prime}=r$. Hence $p^{\prime}$ is a v-line. If $p \subset l$ for some line $l$, then $q \subset l$ for all $q \in \bar{p}$; thus $p^{\prime} \subset l$. This shows that $p^{\prime} \approx p$.

Lemma 3.9. Let $\bar{p}$ be an equivalence class of $v$-lines.

(a) If $q$ and $r$ are nonvoid v-lines in $\bar{p}$, then $q=r$.

(b) If there exists a v-line $q$ in $\bar{p}$ with $q=\varnothing$, then $r=\varnothing$ for all $r \in \bar{p}$.

Proof. (a) Since $q$ and $r$ are both lines, and $\neg(q \neq r)$, it follows from Proposition 2.16 in M07 that $q=r$.

(b) Let $r \in \bar{p}$, and suppose that $r \neq \varnothing$; thus $r$ is a line. Select any two distinct lines; since $q$ is contained in both lines, it follows from Lemma 3.7 that $r$ is contained in both lines, an absurdity. Thus $r=\varnothing$.

\section{EXTENSION POINTS AND EXTENSION LINES}

The e-points and e-lines defined here will be used to construct the projective extension. 
Definition 4.1.

- An extension point, or e-point, is an equivalence class $\bar{\alpha}$ of pencils of lines. The e-points $\bar{\alpha}$ and $\bar{\beta}$ are said to be equal, written $\bar{\alpha}=\bar{\beta}$, if $\alpha \approx \beta$. We say that $\bar{\alpha}$ and $\bar{\beta}$ are distinct, written $\bar{\alpha} \neq \bar{\beta}$, if $\alpha^{\prime} \neq \beta^{\prime}$.

- An extension line, or e-line, is a set $\lambda_{p}$ of e-points, where $p$ is a prime v-line, and where $\bar{\alpha} \in \lambda_{p}$ if $\bar{\alpha}$ satisfies these two conditions:

(1) If $p \neq \varnothing$, then $p \in \alpha^{\prime}$.

(2) If $p=\varnothing$, then $\alpha$ is a parallel pencil.

We say that the e-lines $\lambda_{p}$ and $\lambda_{q}$ are equal, written $\lambda_{p}=\lambda_{q}$, or distinct, written $\lambda_{p} \neq \lambda_{q}$, when $p=q$, or $p \neq q$. The prime v-line $p$ is called the root of $\lambda_{p}$.

- The e-line

$$
\iota=\lambda_{\varnothing}=\{\bar{\alpha}: \alpha \text { is a parallel pencil }\}
$$

will be called the line at infinity.

- An e-point of the form $\overline{Q^{*}}$, where $Q \in \mathscr{P}$, will be said to be a proper e-point. An e-point of the form $\bar{\rho}$, where $\rho$ is a regular pencil, will be called a regular e-point. An e-line of the form $\lambda_{l}$, where $l \in \mathscr{L}$, will be said to be a proper e-line.

\section{Lemma 4.2.}

(a) For any e-points $\bar{\alpha}$ and $\bar{\beta}$, if $\neg(\bar{\alpha} \neq \bar{\beta})$, then $\bar{\alpha}=\bar{\beta}$.

(b) For any e-lines $\lambda_{p}$ and $\lambda_{q}$, if $\neg\left(\lambda_{p} \neq \lambda_{q}\right)$, then $\lambda_{p}=\lambda_{q}$.

Proof. (a) Let $\alpha \subset \rho$, where $\rho$ is a regular pencil. It follows from Lemma 2.13 that $\alpha \approx \rho$ and from Theorem 2.16 that $\alpha^{\prime}=\rho$. Let $l \in \beta$, and suppose that $l \notin \rho$; then $\alpha^{\prime} \neq \beta^{\prime}$, so $\bar{\alpha} \neq \bar{\beta}$, a contradiction. Thus $\neg(l \notin \rho)$, and by Corollary 2.4 we have $l \in \rho$. This shows that $\beta \subset \rho$. Hence $\alpha \approx \beta$, and $\bar{\alpha}=\bar{\beta}$.

(b) The given condition implies that $\neg(p \neq q)$, so $p \approx q$. Since these v-lines are prime, it follows that $p=q$, and hence $\lambda_{p}=\lambda_{q}$.

Lemma 4.3. For any line $l \in \mathscr{L}$,

(a) $\lambda_{l}=\left\{\bar{\alpha}: l \in \alpha^{\prime}\right\}$.

(b) $\lambda_{l} \supset\left\{\overline{Q^{*}}: Q \in l\right\} \cup\left\{\overline{l^{*}}\right\}$.

However, equality in (b) would be constructively invalid.

Proof. Both (a) and (b) are evident. For a Brouwerian counterexample to equality in (b), let $c \in \mathbb{R}$, let $l$ and $m$ be the lines with equations $y=0$ and $y=1-c x$, and construct the pencil $\alpha=\varphi(l, m)$ using Theorem [2.6. Since $l \in \alpha$, we have $\bar{\alpha} \in \lambda_{l}$. If the hypothesis of equality in (b) is applied to the e-line $\lambda_{l}$, then $\bar{\alpha}$ lies in one of the two indicated sets. If $\bar{\alpha}=\overline{l^{*}}$, then $\alpha$ is a parallel pencil, so $c=0$. If $\bar{\alpha}=\overline{Q^{*}}$, where $Q=(d, e)$, then $\alpha \subset Q^{*}$, so $e=0$ and $1-c d=0$; thus $c \neq 0$. Hence LPO results.

The e-point $\overline{l^{*}}$ is called the tip of the e-line $\lambda_{l}$.

Theorem 4.4. An e-line cannot contain fewer than three e-points.

Proof. Assume that an e-line $\lambda_{p}$ contains fewer than three e-points. Suppose that $p \neq \varnothing$; then $p$ is a line, and contains at least two points. It follows from Lemma 4.3 that $\lambda_{p}$ contains at least two proper e-points, and also the e-point $\overline{p^{*}}$, contradicting the assumption. Thus $p=\varnothing$, and $\lambda_{p}$ is the line at infinity $\iota$. Now $\lambda_{p}$ contains the e-points corresponding to three mutually distinct parallel pencils of lines, a final contradiction. 
Lemma 4.5. If $\bar{\alpha}$ and $\bar{\beta}$ are distinct e-points on the line at infinity $\iota$, then $\alpha \cap \beta=\varnothing$. Conversely, if an e-line $\lambda_{p}$ contains distinct e-points $\bar{\alpha}$ and $\bar{\beta}$, where $\alpha$ and $\beta$ are complete pencils with $\alpha \cap \beta=\varnothing$, then $\lambda_{p}=\iota$.

Proof. The first statement follows from Theorem 2.14. For the second statement, first note that it follows from Corollary 2.10 that $\alpha$ and $\beta$ are parallel pencils, and then from Theorem 2.12 that $\alpha^{\prime}$ and $\beta^{\prime}$ are also parallel pencils. Now suppose that $p \neq \varnothing$; then $p \in \alpha^{\prime} \cap \beta^{\prime}$, and it follows from Theorem 2.14 that $\alpha^{\prime} \approx \beta^{\prime}$, so $\bar{\alpha}=\bar{\beta}$, a contradiction. Hence $p=\varnothing$, and $\lambda_{p}=\iota$.

Note on the stem of an e-line. As noted at the beginning of Section 3, the notion of a $v$-line arose in connection with the set of proper e-points that lie on an e-line. For any e-line $\lambda_{p}$, define

$$
s_{p}=\left\{Q \in \mathscr{P}: \overline{Q^{*}} \in \lambda_{p}\right\}
$$

and call this the stem of $\lambda_{p}$.

It is easily seen that $p \subset s_{p}$, that $\neg\left(p \neq s_{p}\right)$, and that $p=s_{p}$ when $p \neq \varnothing$. However, the statement "The stem of any e-line is a $v$-line" is constructively invalid. For a Brouwerian counterexample, let $c \in \mathbb{R}$, and set $q=\{(t, 0): t \in \mathbb{R}$ and $c=$ $0\} \cup\{(0, t): t \in \mathbb{R}$ and $c \neq 0\}$. It is clear that $q$ is a v-line; construct the e-line $\lambda_{p}$ with root $p=q^{\prime}$. To show that $\overline{O^{*}} \in \lambda_{p}$, where $O$ is the origin, note first that the condition $p=\varnothing$ is ruled out by Lemma 3.9. so we need consider only the condition $p \neq \varnothing$. Suppose that $O \notin p$, and suppose further that $c \neq 0$. Then $p=q=m_{0}$, where $m_{0}$ is the $y$-axis, so $O \in p$, a contradiction. Thus $c=0$, and $p=q=l_{0}$, a contradiction. Thus $O \in p$, and $p \in O^{*}$. Since $O^{*}$ is a prime pencil, this shows that $\overline{O^{*}} \in \lambda_{p}$; thus $O \in s_{p}$, so $s_{p}$ is nonvoid. Note that if $c=0$, then $s_{p}=p=q=l_{0}$, while if $c \neq 0$, then $s_{p}=p=q=m_{0}$. By hypothesis, $s_{p}$ is a line; thus either $s_{p} \neq l_{0}$, or $s_{p} \neq m_{0}$. It follows that either $\neg(c=0)$, or $c=0$. Hence WLPO results.

\section{Projective EXtension}

The extension will be constructed and the main projective axioms will be verified.

\section{Definition 5.1.}

- We denote by $\mathscr{P}^{*}$ the family of all e-points, and by $\mathscr{L}^{*}$ the family of all e-lines, retaining the equality and inequality relations, and the relation $\bar{\alpha} \in \lambda_{p}$, adopted in Definition 4.1 .

- For any e-point $\bar{\alpha}$, and any e-line $\lambda_{p}$, we say that $\bar{\alpha}$ lies outside $\lambda_{p}$, written $\bar{\alpha} \notin \lambda_{p}$, if $\bar{\alpha} \neq \bar{\rho}$ for all regular e-points $\bar{\rho}$ in $\lambda_{p}$.

- $\mathscr{G}^{*}=\left(\mathscr{P}^{*}, \mathscr{L}^{*}\right)$ is the projective extension of the incidence plane $\mathscr{G}=(\mathscr{P}, \mathscr{L})$.

Theorem 5.2. Let $\bar{\alpha}$ be any e-point, and $\lambda_{p}$ any e-line. If $\neg\left(\bar{\alpha} \notin \lambda_{p}\right)$, then $\bar{\alpha} \in \lambda_{p}$.

Proof. First let $p \neq \varnothing$, and suppose that $p \notin \alpha^{\prime}$. If $\bar{\rho}$ is any regular e-point on $\lambda_{p}$, then $p \in \rho$, so $\alpha^{\prime} \neq \rho$, and $\bar{\alpha} \neq \bar{\rho}$; this shows that $\bar{\alpha} \notin \lambda_{p}$, a contradiction. Thus we have $\neg\left(p \notin \alpha^{\prime}\right)$, and it follows from Theorem 2.17 that $p \in \alpha^{\prime}$.

Now let $p=\varnothing$; thus $\lambda_{p}=\iota$, the line at infinity. Let $l, m \in \alpha$, and suppose that $l$ intersects $m$. Let $\bar{\rho}$ be any regular e-point on $\lambda_{p}$; then $\rho=n^{*}$ for some line $n$. By Axiom L2 of [M07] we may assume that $n$ intersects $l$, so $l \notin n^{*}$. Thus $\alpha^{\prime} \neq n^{*}$, and $\bar{\alpha} \neq \bar{\rho}$. This shows that $\bar{\alpha} \notin \lambda_{p}$, a contradiction. Thus $l \| m$, and this shows that $\alpha$ is a parallel pencil.

Hence $\bar{\alpha} \in \lambda_{p}$. 
Theorem 5.3. There exists a unique e-line passing through any two distinct epoints.

Proof. (i) Let $\bar{\alpha}$ and $\bar{\beta}$ be distinct e-points; we may assume that $\alpha$ and $\beta$ are complete pencils. By Lemma [3.3, the core $r=\alpha \sqcap \beta$ is a v-line; set $p=r^{\prime}$. First let $p \neq \varnothing$, and assume that $p \notin \alpha^{\prime}$. Suppose that $r \neq \varnothing$; then $p=r \in \alpha$, a contradiction. Thus $r=\varnothing$, so also $p=\varnothing$, a contradiction. This shows that $\neg\left(p \notin \alpha^{\prime}\right)$, and it follows from Theorem 2.17 that $p \in \alpha^{\prime}$. Now let $p=\varnothing$; then also $r=\varnothing$. Since $\alpha$ and $\beta$ are complete pencils, it follows from Corollary 2.10 that $\alpha$ is a parallel pencil. Thus $\bar{\alpha} \in \lambda_{p}$, and similarly $\bar{\beta} \in \lambda_{p}$.

(ii) Let $\lambda_{p}$ and $\lambda_{q}$ be e-lines, each passing through two distinct e-points $\bar{\alpha}$ and $\bar{\beta}$, and assume that $p \neq q$. Suppose that $p \neq \varnothing$; then $\alpha^{\prime} \cap \beta^{\prime}=\{p\}$. Suppose further that $q \neq \varnothing$; then also $\alpha^{\prime} \cap \beta^{\prime}=\{q\}$, so $p=q$, a contradiction. Thus $q=\varnothing$. Now $\alpha$ and $\beta$ are parallel pencils, and so also are $\alpha^{\prime}$ and $\beta^{\prime}$. It follows from Theorem 2.14 that $\alpha^{\prime} \cap \beta^{\prime}=\varnothing$, a contradiction. Thus $p=\varnothing$. Similarly, $q=\varnothing$, so $p=q$, a final contradiction. This shows that $\neg(p \neq q)$, so $p \approx q$. Since $p$ and $q$ are prime v-lines, it follows that $p=q$, and hence $\lambda_{p}=\lambda_{q}$.

Corollary 5.4. Invariance of the core. Let $\lambda_{p}$ be an e-line, and let $\bar{\alpha}$ and $\bar{\beta}$ be any distinct e-points on $\lambda_{p}$, where $\alpha$ and $\beta$ are prime pencils. Then $\alpha \sqcap \beta=p$.

Proof. Set $r=\alpha \sqcap \beta$, and $q=r^{\prime}$. With the construction of Theorem 5.3, $\bar{\alpha}$ and $\bar{\beta}$ will lie on the e-line $\lambda_{q}$. Thus $p=q$; this shows that $r \subset p$. If $Q \in p$, then $p \neq \varnothing$, so $p \in \alpha \cap \beta$. It follows from Theorem 2.12 that $p=r$, and thus $Q \in r$; this shows that $p \subset r$.

Note for Corollary 5.4. A stronger statement, without the condition "prime" pencils, would be constructively invalid. For a Brouwerian counterexample, let $c$ be a real number with $\neg(c=0)$, set $\alpha=(0,0)^{*}$, and set $\beta=\left\{l_{t}: t \in \mathbb{R}\right.$ and $\left.c \neq 0\right\}$. Then $\bar{\alpha}, \bar{\beta} \in \lambda_{l_{0}}$, and $\alpha \sqcap \beta=\{(t, 0): t \in \mathbb{R}$ and $c \neq 0\}$. By hypothesis, we have $\alpha \sqcap \beta=l_{0}$, so $c \neq 0$. Thus MP results.

Classically, the projective axiom concerning a common point for any two lines need be verified only for distinct lines, there being no reason to consider identical lines. Constructively, however, there are always innumerable pairs of lines for which we do not know, at present, whether they are identical or distinct. Thus we require a theorem that deals with two arbitrary lines. The point obtained will be common to the lines in any eventuality, allowing for any possible future discovery that the lines are distinct, or are identical.

Theorem 5.5. Any two e-lines pass through a common e-point. If the e-lines are distinct, then the common e-point is unique.

Proof. Given the e-lines $\lambda_{p}$ and $\lambda_{q}$, construct the complete pencil $\gamma=\varphi(p, q)$, using Theorem 3.4. If $p \neq \varnothing$, then $p \in \varphi_{0}$, so $p \in \gamma \subset \gamma^{\prime}$. If $p=\varnothing$, then it follows from Lemma 3.5 that $\gamma$ is a parallel pencil. Hence $\bar{\gamma} \in \lambda_{p}$, and similarly $\bar{\gamma} \in \lambda_{q}$. Thus $\bar{\gamma}$ is a common e-point. If $\lambda_{p} \neq \lambda_{q}$, then uniqueness of the common e-point follows from Theorem 5.3 and Lemma 4.2 .

Note on cotransitivity. The Brouwerian counterexample in [M13] shows that in any projective-type extension, cotransitivity for points is constructively incompatible with the existence of a common point for any two lines. It follows that the 
statement "In the extension $\mathscr{G}^{*}$, the cotransitivity property holds for e-points" is constructively invalid.

For a direct Brouwerian counterexample, using the constructions of $\mathscr{G}^{*}$, let $c \in \mathbb{R}$, set $d=\max \{c, 0\}$, set $e=\max \{-c, 0\}$, let $l$ be the line on $\mathbb{R}^{2}$ with equation $y=1-d x$, let $m$ be the line with equation $x=1-e y$, set $\alpha=\varphi\left(l_{0}, l\right)$, set $\beta=\varphi\left(m_{0}, m\right)$, where $m_{0}$ is the $y$-axis, set $p=\{(1 / c, t): t \in \mathbb{R}$ and $c>0\}$, set $q=\{(t, 1 / c): t \in \mathbb{R}$ and $c<0\}$, and set $\gamma=\varphi(p, q)$ using Theorem 3.4. To show that $\bar{\alpha} \neq \bar{\beta}$, it will suffice to show that $l_{0} \notin \beta^{\prime}$. For any line $n \in \beta^{\prime}$, it follows from Axiom L2 in [M07] that either $n$ intersects $l_{0}$ or $n$ intersects $m_{0}$. In the first case, $n \neq l_{0}$. In the second case, say $n \times m_{0}=(0, h)$; then $\beta^{\prime}=(0, h)^{*}$. Since $m \in \beta$, we have $0=1-e h$, so $h \neq 0$, and thus $(0, h) \notin l_{0}$; it follows that $n \neq l_{0}$. This shows that $l_{0} \notin \beta^{\prime}$. Thus $\alpha^{\prime} \neq \beta^{\prime}$, and $\bar{\alpha} \neq \bar{\beta}$. By hypothesis, we have either $\bar{\gamma} \neq \bar{\alpha}$ or $\bar{\gamma} \neq \bar{\beta}$. In the first case, suppose that $c<0$. Then $d=0$, so $\alpha=l_{0}^{*}$. Also, $p=\varnothing$, and $q=l_{1 / c}$, so $\gamma=q^{*}=l_{0}^{*}=\alpha$, a contradiction; thus $c \geq 0$. Similarly, in the case $\gamma \neq \bar{\beta}$ we find that $c \leq 0$. Hence LLPO results.

For an alternative counterexample, use the v-line $p=\{(t, 0): t \in \mathbb{R}$ and $c=$ $0\} \cup\{(0, t): t \in \mathbb{R}$ and $c \neq 0\}$ to construct the pencil $\gamma=\varphi(p, p)$. Assuming cotransitivity for e-points, we have either $\bar{\gamma} \neq \overline{l_{0}^{*}}$ or $\bar{\gamma} \neq \overline{m_{0}^{*}}$, where $m_{0}$ is the $y$-axis. Hence WLPO results.

Cotransitivity for e-lines in the extension $\mathscr{G}^{*}$ is also constructively invalid; this may be seen using the counterexample at the end of Section 4

Theorem 5.6. The projective plane $\mathscr{G}^{*}=\left(\mathscr{P}^{*}, \mathscr{L}^{*}\right)$ is an extension of the incidence plane $\mathscr{G}=(\mathscr{P}, \mathscr{L})$.

Proof. Set $\mathscr{P}^{\prime}=\left\{\overline{Q^{*}}: Q \in \mathscr{P}\right\}$, the family of proper e-points, and set $\mathscr{L}^{\prime}=\left\{\lambda_{l}\right.$ : $l \in \mathscr{L}\}$, the family of proper e-lines. Then the mappings $Q \mapsto \overline{Q^{*}}(Q \in \mathscr{P})$ and $l \mapsto \lambda_{l}(l \in \mathscr{L}) \operatorname{map} \mathscr{G}$ into $\mathscr{G}^{*}$, with image $\mathscr{G}^{\prime}=\left(\mathscr{P}^{\prime}, \mathscr{L}^{\prime}\right)$.

If $Q=R$, then clearly $\overline{Q^{*}}=\overline{R^{*}}$. Conversely, if $\overline{Q^{*}}=\overline{R^{*}}$, then $Q^{*} \approx R^{*}$, and since these pencils are regular, we have $Q^{*}=R^{*}$. Select distinct lines $l$ and $m$ in $Q^{*}$; thus $l \times m=Q$. Since also $l, m \in R^{*}$, we have $l \times m=R$, so $Q=R$.

If $Q \neq R$, we may select a line $l$ so that $Q \in l$ but $R \notin l$. Thus $l \in Q^{*}$, but $l \notin R^{*}$, so $Q^{*} \neq R^{*}$; since these pencils are prime, we have $\overline{Q^{*}} \neq \overline{R^{*}}$. Conversely, if $\overline{Q^{*}} \neq \overline{R^{*}}$, then $Q^{*} \neq R^{*}$, and we may select a line $m \in Q^{*}$ with $m \notin R^{*}$. Then $Q \in m$ but $R \notin m$, so $Q \neq R$.

For any lines $l$ and $m$, it follows from Definition 4.1 that $l=m($ or $l \neq m$ ) if and only if $\lambda_{l}=\lambda_{m}$ (or $\lambda_{l} \neq \lambda_{m}$ ). Thus the mappings preserve the equality and inequality relations for points and lines.

Clearly, $Q \in l$ if and only if $\overline{Q^{*}} \in \lambda_{l}$. Now let $Q \notin l$. For any e-point $\bar{\beta} \in \lambda_{l}$, we have $l \in \beta^{\prime}$, but $l \notin Q^{*}$; thus $Q^{*} \neq \beta^{\prime}$, so $\overline{Q^{*}} \neq \bar{\beta}$. Thus $\overline{Q^{*}} \notin \lambda_{l}$. Conversely, let $\overline{Q^{*}} \notin \lambda_{l}$. For any point $R \in l$, we have $\overline{R^{*}} \in \lambda_{l}$; thus $\overline{Q^{*}} \neq \overline{R^{*}}$, so $Q \neq R$. Thus $Q \notin l$. Thus the mappings preserve the relations point on a line and point outside a line.

Hence the mappings $Q \mapsto \overline{Q^{*}}$ and $l \mapsto \lambda_{l}$ form a strict isomorphism of $\mathscr{G}$ onto $\mathscr{G}^{\prime}$, a sub-plane of $\mathscr{G}^{*}$.

\section{REFERENCES}

[B65] E. Bishop, Book Review: The Foundations of Intuitionistic Mathematics, by S. C. Kleene and R. E. Vesley. Bull. Amer. Math. Soc. 71 (1965), no. 6, 850-852. MR1566375 
[B67] - Foundations of Constructive Analysis. McGraw-Hill Book Co., New YorkToronto-London, 1967. MR0221878 (36:4930)

[BB85] E. Bishop and D. Bridges, Constructive Analysis. Springer-Verlag, Berlin, 1985. MR.804042 (87d:03172)

[BR87] D. Bridges and F. Richman, Varieties of Constructive Mathematics. Cambridge University Press, Cambridge, 1987. MR890955 (88k:03127)

[Br08] L. E. J. Brouwer, De onbetrouwbaarheid der logische principes. Tijdschrift voor Wijsbegeerte 2 (1908), 152-158. English translation, "The Unreliability of the Logical Principles", pp. 107-111, in Heyting, A. (ed.), L. E. J. Brouwer: Collected Works 1: Philosophy and Foundations of Mathematics. Amsterdam-New York, Elsevier, 1975.

[D63] D. van Dalen, Extension problems in intuitionistic plane projective geometry I, II, Indag. Math. 25 (1963), 349-383. MR0153567(27:3531a)

[H59] A. Heyting, Axioms for intuitionistic plane affine geometry, in L. Henkin, P. Suppes, A. Tarski (eds.), The Axiomatic Method, with special reference to geometry and physics: Proceedings of an international symposium held at the University of California, Berkeley, December 26, 1957 - January 4, 1958. North-Holland, Amsterdam, 1959, 160-173. MR0120154(22:10911)

[M83] M. Mandelkern, Constructive Continuity. Memoirs Amer. Math. Soc. 42 (1983), nr. 277. $\operatorname{MR} 690901$ (84j:26007)

[M85] , Constructive mathematics, Math. Mag. 58 (1985), 272-280. MR810148 (87b:03001)

[M88] , Limited omniscience and the Bolzano-Weierstrass principle, Bull. London Math. Soc. 20 (1988), 319-320. MR940284 (89e:03108)

[M89] _ Brouwerian counterexamples, Math. Mag. 62 (1989), 3-27.

[M07] - Constructive coordinatization of Desarguesian planes, Beiträge Algebra Geom. 48 (2007), 547-589.

[M13] The common point problem in constructive projective geometry, Indag. Math. (N.S.) 24 (2013), no. 1, 111-114. MR2997755

[R82] F. Richman, Meaning and information in constructive mathematics, Amer. Math. Monthly 89 (1982), 385-388. MR660918 (83f:03006)

[R02] - Omniscience principles and functions of bounded variation, Math. Logic Quart. 42 (2002), 111-116. MR 1874208 (2002i:03080)

[S70] G. Stolzenberg, Review of E. Bishop, Foundations of Constructive Analysis, Bull. Amer. Math. Soc. 76 (1970), 301-323. MR0257687(41:2337)

\footnotetext{
Department of Mathematics, New Mexico State University, Las Cruces, New Mexico 88003

E-mail address: mandelkern@member.ams.org
} 